\title{
Modelling of platelet aggregation in aneurysm
}

\author{
K. Shimano ${ }^{1}$, T. Hayashi ${ }^{1}$, H. Ujiie ${ }^{2}$, T. Ono ${ }^{1} \&$ Y. Enomoto ${ }^{1}$ \\ ${ }^{I}$ Department of Mechanical Systems Engineering, \\ Musashi Institute of Technology, Japan \\ ${ }^{2}$ Tokyo Women's Medical University, Japan
}

\begin{abstract}
Thrombi are often found in aneurysms and are considered to play an important role in rupture. It is crucial to scrutinise any correlation between the probability of rupture and the extent to which thrombi are generated in the aneurysm. Numerical techniques such as computational fluid dynamics (CFD) are promising tools in the biomedical field. However, there are, at present, no models that allow us to evaluate thrombus generation. The authors aim at the proposal of such a model. In the present study, the process of platelet aggregation is considered. In blood flow near the entry to an aneurysm, red blood cells are haemolysed due to high shear stress or high pressure. The ensuing release of adenosine diphosphate (ADP) induces the aggregation. Making reference to actual aggregation curves of human plasma for various ADP concentrations, the authors have modelled the rate at which the density of aggregated platelets continues to increase in the aggregation process. A combination of CFD and the present model enables us to obtain the distribution of platelets clotting in an aneurysm.
\end{abstract}

Keywords: computational fluid dynamics, platelet aggregation, aneurysm.

\section{Introduction}

Rupture of a cerebral artery aneurysm causes a life-threatening subarachnoid haemorrhage. Although not all aneurysms rupture, it is difficult to predict which aneurysms are likely to do so. Ujiie et al. [1] reported a significant correlation between the probability of rupture and aspect ratio of the aneurismal dome depth to the neck width: almost $80 \%$ of ruptured aneurysms among those investigated by them had aspect ratio more than 1.6. According to a theory proposed by Ujiie et al. [2], thrombi are likely to form in an aneurysm of high aspect ratio due to 
considerably slow blood flow in it. Ensuing fibrinolysis in which thrombi are dissolved is considered to lead to rupture because the fibrinolysis can cause damage to the endothelium. In other words, blood flow and thrombus formation in the aneurysm are presumably the greatest factors in the probability of rupture. Takahashi et al. [3] showed evidence supporting the hypothesis. In their study, canine blood flow in endothelialised glass aneurysm models was visualised and thrombus formation was observed for a large value of the aspect ratio.

For further progress in the area of the rupture prediction, more understanding of the flow and the thrombus formation in aneurysms is essential. Approaches based on numerical techniques such as computational fluid dynamics (CFD) are promising for this purpose. However, there are, at present, no models that allow us to evaluate thrombus formation. The authors aim at the proposal of such a model and, in the present paper, the process of platelet aggregation is modelled as the first step.

Adenosine diphosphate (ADP) released from haemoglobin as a result of haemolysis is considered to induce the platelet aggregation in an aneurysm. Therefore, an ADP concentration is the primary parameter in the present aggregation model. Making reference to actual aggregation curves of human plasma for various ADP concentrations, processes of the first and the second aggregations are modelled. Dissociation of the first aggregation and positive feedback caused by granule release at the second aggregation are also taken into account. Details of the present model are discussed in Section 2.

Unsteady blood flow in one of the glass aneurysm models tested by Takahashi et al. [3] is simulated by the authors' CFD code based on the finite volume method and the unstructured grid. A combination of the present aggregation model and the flow information provided by CFD reveals the distribution of platelets clotting in the glass aneurysm model. The numerical techniques used are described in Section 3 and the computational results are presented in Section 4.

\section{Aggregation model}

\subsection{Modelling framework}

In blood flow near the entry to an aneurysm, red blood cells are haemolysed due to high shear stress or high pressure. The following release of ADP induces the aggregation. The process of the aggregation consists of two stages. In the first stage, platelets are activated and begin to adhere to each other (the first aggregation). Some of those activated platelets return to their original inactive state because their bonds at this stage are not sufficiently strong. This is called dissociation. On the other hand, those that remain active proceed to the second stage with releasing granules into the plasma (the second aggregation). Platelets reaching this point can no longer return to the prior inactive state. ADP originating from the released granules contributes to activation of still more platelets. In order to express these phenomena quantitatively, source terms of the aggregations are expressed as eqns (1) and (2): 


$$
\begin{gathered}
\dot{N}_{1}=S_{N 01}-S_{N 12}-S_{N 10} \\
\dot{N}_{2}=S_{N 12}
\end{gathered}
$$

where $N_{1}$ and $N_{2}$ represent densities of platelets in the first and the second aggregations, respectively. Eqn (1) includes three source terms, $S_{N 01}, S_{N 12}$ and $S_{N 10}$. The first source term $S_{N 01}$ represents the rate of change from the inactive state to the first aggregation while the third term $S_{N 10}$ denotes the rate of the dissociation. The second term $S_{N 12}$ means the rate of progress to the second stage and appears also in eqn (2).

The goal of the modelling is to determine these three source terms so that the system of equations is closed. It is assumed in this study that the source terms should depend on three parameters: $N_{1}, N_{2}$ and $n_{A}$. Here, $n_{A}$ is a concentration of ADP. By this choice, it is also necessary to include $S_{A}$, the source term of ADP, in the modelling framework.

\subsection{Modelling of source terms}

According to Folie and McIntire [4], the ADP generation by the release of dense granules is proportional to the growth rate of the second aggregation:

$$
S_{A}=K_{A} \cdot S_{N 12} \text {. }
$$

The authors follow this strategy and use the same value for the constant $K_{A}$ as in Folie and McIntire [4]. Eqn (3) leads to the relation between $n_{\mathrm{A}}$ and $N_{2}$ :

$$
n_{A}=K_{A} \cdot N_{2}+n_{A 0} \text {. }
$$

Eqn (4) is of great importance because it reduces the number of variables from three to two: only $N_{1}$ and $N_{2}$ should be considered for a fixed initial ADP concentration $n_{\mathrm{A} 0}$. Note that eqn (4) represents a plane in the three dimensional space $\left(N_{1}, N_{2}, n_{A}\right)$.

For a certain value of $n_{\mathrm{A} 0}$, the percentage of aggregated platelets changes with time. This relation is clinically obtained as an aggregation curve. In modelling the source terms, the authors make reference to actual aggregation curves of human plasma. An example of aggregation curves is depicted in fig. 1 by a solid line. Generally, an aggregation curve only provides information about the total amount of aggregated platelets, namely $N_{1}+N_{2}$. A gradient of the aggregation curve is equivalent to $S_{N 01}-S_{N 10}$ because eqns (1) and (2) show

$$
\dot{N}_{1}+\dot{N}_{2}=S_{N 01}-S_{N 10} \text {. }
$$

The dissociation term $S_{N 10}$ is expressed by

$$
S_{N 10}=\frac{\ln 2}{T_{d}} \cdot N_{1} .
$$

where $T_{d}$ is a constant similar to a half-life period. In the present model, $T_{d}$ $=10\left[\mathrm{sec}\right.$.]. This is determined by trial and error. $S_{N 01}$ can be evaluated by adding $S_{N 10}$ in (6) to the gradient of the original aggregation curve.

The model for the source term $S_{N 12}$ is given by eqn (7).

$$
S_{N 12}=\lambda N_{1}\left(1-\frac{N_{2}}{N_{2 \max }}\right) \text {. }
$$


This equation is based on several facts and physical intuitions related to the second aggregation. Firstly, the irreversibility of the second aggregation ensures monotone increase of $N_{2}$ and positive $S_{N 12}$. Secondly, $N_{2}$ should be bounded from above because $N_{2}$ never increases boundlessly. From these two facts, $N_{2}$ is expected to asymptotically approach a certain value $N_{2 \max }$, which depends on the initial ADP concentration $n_{A 0}$. As a result, the source term $S_{N 12}$ should approach zero as $N_{2}$ becomes close to $N_{2 \max }$. There is another fact that only platelets in the first stage of the aggregation can proceed to the second stage. This gives an intuition that the source term $S_{N 12}$ is proportional to $N_{1}$. Finally, $\lambda$ is a constant with a dimension of [1/time]. Here, $\lambda$ is chosen to be $1 / 40[1 / \mathrm{sec}$.] because the time scale of the aggregation seems approximately 40seconds from the shape of aggregation curves.

Using the model discussed above, $N_{1}$ and $N_{2}$ can be calculated separately. The result for $N_{1}$ is plotted in fig. 1 by a dotted curve and that for $N_{2}$ by dashed one.

A path of the aggregation can be drawn on the $\left(N_{1}, N_{2}\right)$ plane. An example of such paths is shown by a thick curve in fig. 2 for $n_{\mathrm{A} 0}=5 \mu \mathrm{M}$. In fig. $2, N_{1}$ and $N_{2}$ are expressed as percentages to the total platelet density $N_{\text {plt }}$.

If the present modelling strategy is applied repeatedly to various initial ADP concentrations, corresponding paths will be obtained and their union will form a curved surface in the $\left(N_{1}, N_{2}, n_{A}\right)$ space. This surface is called "the aggregation surface" in this paper. A section of the aggregation surface by the plane (4) for $n_{\mathrm{A} 0}=5 \mu \mathrm{M}$ agrees exactly with the thick curve in fig. 2 .

Values of all the source terms in eqns (1) and (2) are available for any set of $\left(N_{1}, N_{2}, n_{A}\right)$ on the aggregation surface. In practice, due to limited variation of initial ADP concentrations, interpolations are used to form the whole aggregation surface. In this study, the authors refer to aggregation curves for $n_{\mathrm{A} 0}=1,2,5$ and $10 \mu \mathrm{M}$ obtained by Yazuka et al. [5].

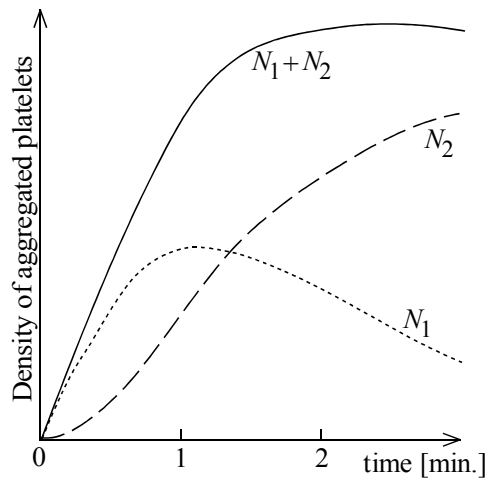

Figure 1: Example of aggregation curves and separation of $N_{1}$ and $N_{2}$.

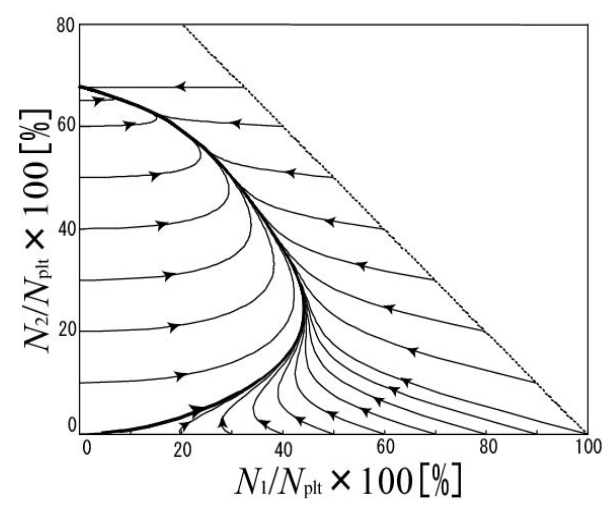

Figure 2: Aggregation path and auxiliary paths representing off-surface behaviour for $n_{\mathrm{A} 0}=5 \mu \mathrm{M}$. 


\subsection{Behaviours off the aggregation plane}

In real blood flow, the set $\left(N_{1}, N_{2}, n_{A}\right)$ may deviate from the aggregation surface due to effects of convection and diffusion. Off-surface behaviours must be determined so that the model returns values of the source terms for an arbitrary set of $\left(N_{1}, N_{2}, n_{A}\right)$.

For $S_{N 10}$ and $S_{N 12}$, it is assumed that eqns (6) and (7) hold. On the other hand, $S_{N 01}$ is considered to be always under the strong influence of $n_{A}$ and also that of $N_{2}$ through eqn (4). Thus, if the set $\left(N_{1}, N_{2}, n_{A}\right)$ is off the aggregation surface, $S_{N 01}$ for the corresponding set $\left(N_{1}{ }^{\prime}, N_{2}, n_{A}\right)$ located on the surface is adopted. In addition, a linear damping function is multiplied to $S_{N 01}$ for a too large value of $N_{1}$. Thin curves in fig. 2 are examples of auxiliary paths representing the offsurface behaviours.

\subsection{Comments}

It is obvious from aggregation curves that conditions necessary for aggregation are not only a high ADP concentration but also a long residence time. It might seem that time should be included in an aggregation model as an independent variable. However, from a practical point of view, time is a wrong choice because, in many CFD applications, governing equations are based on the eularian method of description in which a history of an individual fluid particle is not concerned. It is difficult to define the origin of time $(t=0)$ unless the lagrangian approach is adopted. It is remarkable that, in the present model designed to fit the eularian approach, the combination of $N_{1}$ and $N_{2}$ implicitly expresses what stage the aggregation is currently at.

\section{Application of aggregation model}

In combination with CFD, the present aggregation model is applied to one of glass aneurysm models tested by Takahashi et al. [3]. The glass model imitates an aneurysm at a cerebrovascular branch and its aspect ratio exceeds 1.6: the depth of the aneurysm is approximately $6.5 \mathrm{~mm}$ while the neck width is $2.8 \mathrm{~mm}$. The inner diameter of each artery is $2 \mathrm{~mm}$.

The aggregation model is applied after the flow field is calculated by CFD because the flow velocity is necessary in evaluation of the convection effect.

\subsection{Calculation of blood flow}

The unsteady incompressible Navier-Stokes equations are numerically solved. Canine blood is assumed to be Newtonian fluid. The flow rate changing cyclically with $0.28 \mathrm{sec}$. period is imposed at the vascular inlet as a boundary condition. The Reynolds number based on the average inlet velocity and the inner diameter of the vascular ranges from 244 to 485 and the flow is treated as laminar.

The cell-centred finite volume approach is used for spatial discretisation of the governing equations with 886893 unstructured computational cells. Most of 
the cells are tetrahedra. Also hexahedra and trigonal prisms are allocated along the wall surface of the aneurysm so that deterioration in the accuracy at the boundary layer can be avoided. The height of computational cells distributed on the aneurismal wall is so small as $0.06 \mu \mathrm{m}$. For reduction of computational time, the Navier-Stokes solver is parallelised by domain decomposition and implemented on a PC cluster with eight Pentium-4 processors. MPI (message passing interface) is used in parallelism.

The time increment $\Delta t$ is $0.0001 \mathrm{sec}$. The SIMPLE algorithm is employed for coupling of pressure and velocity. Calculation is terminated when sufficient periodicity in the simulated flow is observed.

\subsection{Calculation of scalars in aggregation model}

The model explained in Section 2 only describes source terms related to the aggregation. As, in real blood, there are effects of convection and diffusion, transport equations for $N_{1}, N_{2}$ and $n_{A}$ are solved. The generalised form of the transport equations is expressed as follows:

$$
\frac{\partial \phi}{\partial t}+\frac{\partial(u \phi)}{\partial x}+\frac{\partial(v \phi)}{\partial y}+\frac{\partial(w \phi)}{\partial z}=\frac{\partial}{\partial x}\left(\Gamma_{\phi} \frac{\partial \phi}{\partial x}\right)+\frac{\partial}{\partial y}\left(\Gamma_{\phi} \frac{\partial \phi}{\partial y}\right)+\frac{\partial}{\partial z}\left(\Gamma_{\phi} \frac{\partial \phi}{\partial z}\right)+S_{\phi}
$$

where $\Gamma_{\phi}$ and $S_{\phi}$ represent the diffusion coefficient and the source term of quantity $\phi$, respectively.

The transport equations for $N_{1}, N_{2}$ and $n_{A}$ are solved after the flow field is obtained by CFD because the flow velocity is necessary for evaluation of the convection. Strictly, a mass of aggregated platelets is a solid interacting with the flow field. However, in this study, $N_{1}$ and $N_{2}$ are so small that they are treated as passive scalars with no influence on the flow. The diffusion coefficients of $N_{1}$ and $N_{2}$ are chosen to be zero.

The same computational cells as in CFD analysis are used for solutions of the scalar transport equations while the time increment $\Delta t$ for these scalars is $0.0005 \mathrm{sec}$. Time-marching is conducted by an implicit scheme with the SOR method as an iterative solver. Calculation is terminated after 10 cycles of the pulsebeat (approximately $2.8 \mathrm{sec}$.). Therefore, the set $\left(N_{1}, N_{2}, n_{A}\right)$ stays in the vicinity of the $n_{A}$-axis in the $\left(N_{1}, N_{2}, n_{A}\right)$ space. The transport equation solver is parallelised in the same manner as the $\mathrm{N}-\mathrm{S}$ solver.

In addition to the ADP source term $S_{A}$ defined by eqn (3), ADP generation by haemolysis should be taken into consideration. There are no haemolysis models available at present. Here, for simplicity, it is assumed that $20 \%$ of red blood cells impinging on the neck of the aneurysm are haemolysed.

\section{Computational results}

Figure 3 shows velocity vectors on the central cross-sectional plane at diastolic and systolic phases. It is clearly observed that blood flow hits the left edge of the aneurismal neck. Not only at the timings shown in fig. 3, blood flow is always impinging on the left edge of the neck. This causes haemolysis. After this impingement, some blood containing ADP goes into the dome with changing its 
direction to the right and impinges again on the wall of the right hemisphere of the aneurysm. This is not expected to haemolyse red blood cells because it is much weaker than the first impingement on the neck. The spot of the second impingement depends on time. At the higher flow rate shown in fig.3(b), the stronger inertia makes the flow proceed deeper into the dome.

As a result of the second impingement, the flow spreads three-dimensionally with reducing its speed. Although only blood flowing upward is clearly seen in fig. 3, some blood moves to the span-wise directions. This motion of the blood forms a low-speed region, which occupies a large part in the dome. This region filled with curved path lines of the blood seems identical to the vortex reported by Takahashi et al. [3]. It is noteworthy that the blood motion in the dome after the second impingement is so slow that there is a fairly long time for platelets to aggregate.

The blood moving slowly in the dome turns downward and goes out of the dome through the neck. This motion is hardly observed in fig. 3 because the incoming flow through the neck is dominant on the central plane.

Distribution of $N_{2}$ calculated by the present aggregation model is shown in fig. 4. Each image in fig. 4 represents the density of 2 nd-aggregated platelets in the vicinity of the wall surface at the corresponding timing.

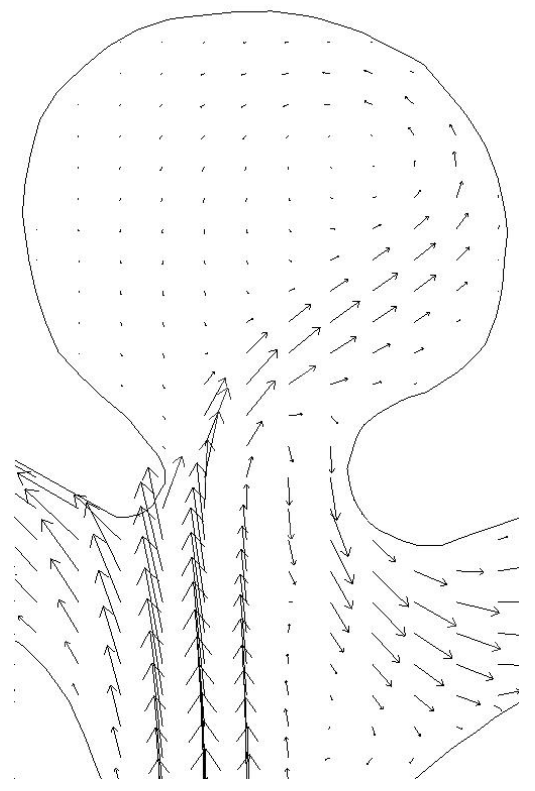

(a)

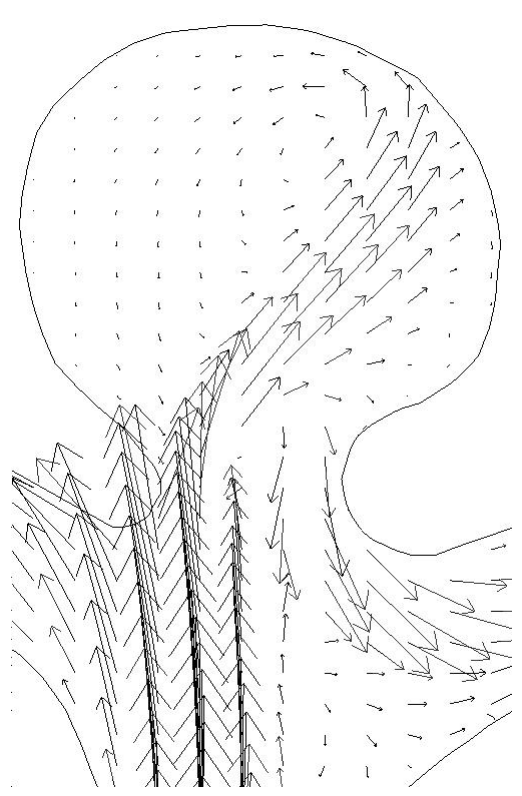

(b)

Figure 3: Velocity vectors on the central cross section (a) at a diastolic phase and (b) at a systolic phase. 


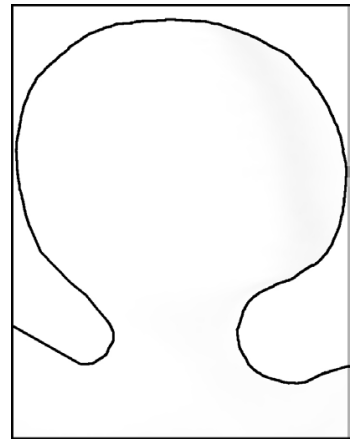

(a) after 2 cycles

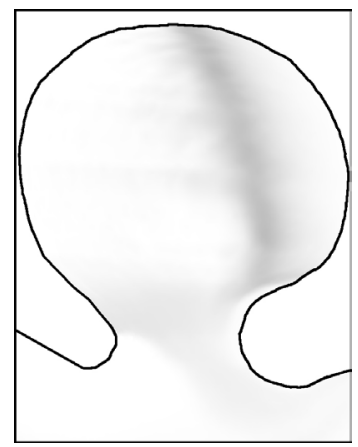

(d) after 5 cycles

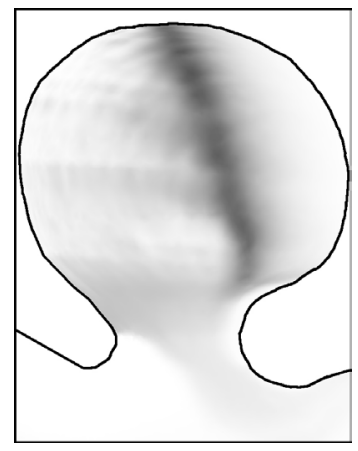

(g) after 8 cycles

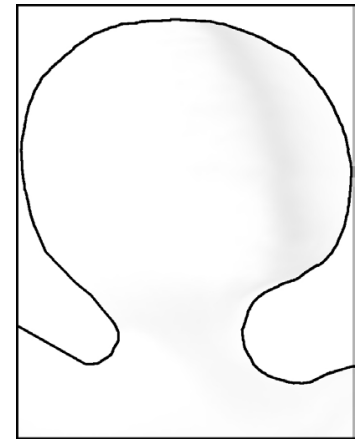

(b) after 3 cycles

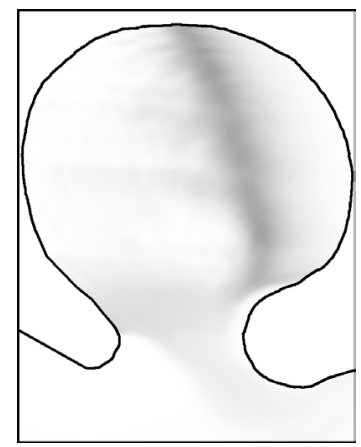

(e) after 6 cycles

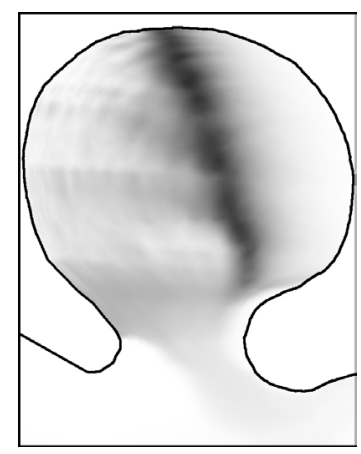

(h) after 9 cycles

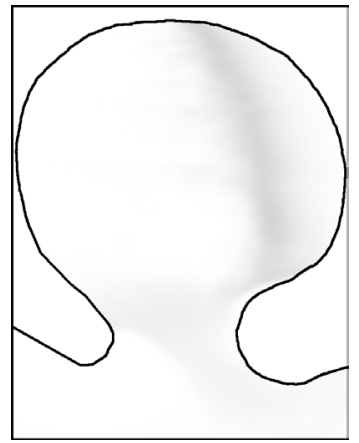

(c) after 4 cycles

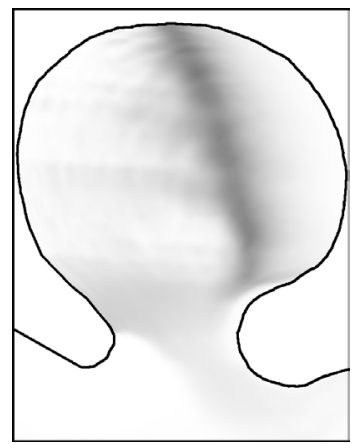

(f) after 7 cycles

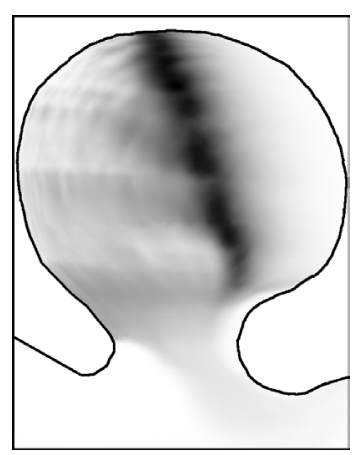

(i) after 10 cycles

Figure 4: Distribution of density of 2nd-aggregated platelets in the vicinity of the wall surface.

A circular belt-shaped region of large $N_{2}$ is clearly observed in fig. 4. This region appears in the right hemisphere at the early stage and moves slowly to the left with $\mathrm{N}_{2}$ increasing. The movement of the region is governed by the blood flow after the second impingement. As the circular region moves to the left, the 
centre of the circular sweeps out a straight line of which intersection with the dome wall almost agree with the average spot of the second impingement.

The maximum values of $N_{2}$ amounts to $0.08 \%$ of the whole platelets after 10 pulsebeat cycles (fig. 4 (i)) and continues to increase afterwards. $N_{1}$ shows similar distribution near the wall with a peak value of $3 \%$, approximately 40 times larger than that of $N_{2}$.

The distribution pattern of $N_{1}$ and $N_{2}$ explained above is limited to the vicinity of the wall where flow is considerably slow. For example, in the belt-shaped region after 10 cycles, all components of flow velocity are on a level of $0.1 \mathrm{~mm} / \mathrm{sec}$ or less. A large value of $N_{1}$ or $N_{2}$ is impossible at a place far from the dome wall where blood moves at a higher speed because the fluid containing a large amount of ADP is immediately swept away. It should be recalled that conditions necessary for aggregation are not only a high ADP concentration but also a long residence time. The present model succeeds in representing this characteristic of the platelet aggregation.

Higher accuracy in geometric modelling may be required because surface roughness of the computational model seems to affect the computational results: a striped pattern in the $N_{2}$ distribution is found in fig.4. In this study, the 3-d computational model was made from several digital images of the glass aneurysm. A more sophisticated modelling technique should be adopted.

Takahashi et al. [3] reported a large amount of thrombus was found near the right edge of the neck. The present result that $N_{1}$ and $N_{2}$ are large near the wall in the right hemisphere may be supported by this fact. However, the region of large $N_{1}$ and $N_{2}$ does not agree precisely with that reported by Takahashi et al. Further verification of the present aggregation model is necessary. Neither coagulation nor adhesion to endothela is considered in this study. Models for these two important phenomena are essential for accurate prediction of the thrombus formation.

\section{Conclusions}

The authors proposed a model of platelet aggregation in aneurysms making reference to actual aggregation curves. Time is not included in the model: instead, the combination of $N_{1}$ and $N_{2}$ implicitly represents the current stage of aggregation.

The present aggregation model was applied to blood flow in a glass aneurysm model in combination with CFD analysis. A region of the high density of aggregated platelets was observed near the wall in the right hemisphere of the aneurysm. Blood flow in this region was very slow. It can be concluded that the present aggregation model reflects a characteristic that a long residence time of ADP is necessary for aggregation.

\section{Acknowledgements}

The authors would like to thank Mr Noriyoshi Takahashi from Tokyo Science University for supplying important information about his experimental study. 
Thanks are also due to Mr Shun Yonezu who assisted the authors in visualisation of the computational results.

\section{References}

[1] Ujiie, H., Tamano, Y., Sasaki, K. \& Hori, T., Is the aspect ratio a reliable index for predicting the rupture of a saccular aneurysm? Neurosurgery, 48(3), pp.495-503, 2001.

[2] Ujiie, H., Tachibana, H., Hiramatsu, O., Hazel, A.L., Matsumoto, T., Ogasawara, Y., Nakajima, H., Hori, T., Takakura, K. \& Kajiya, F., Effects of size and shape (aspect ratio) on the hemodynamics of saccular aneurysms: a possible index for surgical treatment of intracranial aneurysms. Neurosurgery, 45(1), pp.110-130, 1999.

[3] Takahashi, N., Ujiie, H., Yotoriyama, T., Suzuki, Y., Hori, T. \& Kaibara, M., Flow visualization study of the endothelialized glass aneurysm model implanting canine carotid artery (in Japanese with English abs.), J. Jpn. Soc Biorheol., 18(4), pp.143-148, 2004.

[4] Folie, B.J. \& McIntire, L.V., Mathematical analysis of mural thrombogenesis: Concentration profiles of platelet-activating agents and effects of viscous shear flow, Biophys. J., 56, pp.1121-1141, 1989.

[5] Yazuka, C., Houjyo, E. \& Mori, M., Evaluation for platelet aggregation in various condition (in Japanese), Saitama J. Med. Tech., 41(3), pp.219223, 1994. 